\section{SOI: $\underline{1.1 / \mathrm{TAS}}$ DOI: $\underline{10.15863 / \mathrm{TAS}}$ \\ International Scientific Journal Theoretical \& Applied Science}

p-ISSN: 2308-4944 (print) $\quad$ e-ISSN: 2409-0085 (online)

Year: 2015 Issue: 03 Volume: 23

Published: $30.03 .2015 \quad$ http://T-Science.org
Qassim Shamkhi AL-Khafaji

Assistant Professor

Department of Physics, Faculty of Science, University of Kufa,

Najaf, Iraq

qasimsh.alkhafaji@uokufa.edu.iq

SECTION 3. Nanotechnology. Physics.

\title{
COMPUTATION THE HARTREE-FOCK ENERGY OF GROUND STATE FOR BORON ATOM AND ISO ELECTRONIC SERIES
}

Abstract: Partition method have been used to calculate the Hartree-Fock energy of ground state for Boron atom and like ions such as $C+1$ and N+2 in addition to study some important atomic properties using Hartree Fock approximation in position space. Finally all the studied properties are calculated using atomic units.

Key words: Hartree- Fock approximation, position space, Atomic properties, Electronic series.

Language: English

Citation: AL-Khafaji QS (2015) COMPUTATION THE HARTREE-FOCK ENERGY OF GROUND STATE

FOR BORON ATOM AND ISO ELECTRONIC SERIES. ISJ Theoretical \& Applied Science 03 (23): 18-24.

Soi: http://s-o-i.org/1.1/TAS*03(23)4 Doi: crossef http://dx.doi.org/10.15863/TAS.2015.03.23.4

\section{Introduction}

Hartree-fock atomic wave function are independent-particle-model approximations to the nonrelativistic Schrodinger's equation for stationary states . the use of slater determinants accounts for the Pauli principle ,and for an N-electron system the HF equations yield $\mathrm{N}$ Hartree -Fock spin orbitals. In conventional Hartree-Fock calculation, the spin orbitals are expressed as products of a radial function times spherical harmonic times a spin function, the radial functions are taken to depend only on the quantum numbers $\boldsymbol{n}$ and $\boldsymbol{\ell}$, and the total wave function is required to be an Eigen function of the total orbital and spin angular momentum; the form of the spin orbitals guarantees that $\boldsymbol{L}_{z}, \boldsymbol{S}_{z}$, and parity are good quantum numbers. Conventional HF is also known as restricted HF [1].

Schrödinger equation was formulated by the Austrian physicist Erwin Schrödinger it describes how the quantum states of a physical systems changes in time. It is also called wave function an state vector [2]. The wave function $\Psi$, sometimes known as the state function of a system is a function of each particles coordinates (position and spin) and time, it provides a complete description of quantum system [3]. It is well known that the computation of atomic and molecular multielectron properties requires solutions of the Schrödinger equation more accurate than the results obtained from the hartreefock (HF) equation. The variation method for improving the HF solution in which the interelectronic coordinates are explicitly included in the wave function was first introduced by Hylleraas [4]. The Hartree-Fock (HF) method gives roughly $99 \%$ of the total electronic energy where ignores the correlation between electronic [5].

\section{Theory}

The two-particle radial density distribution function $D\left(r_{1}, r_{2}\right)$, is the measure of probability of finding two-electrons simultaneously and their radial coordinates are in the range $r_{1}$ to $r_{1}+d r_{1}$, and $r_{2}$ to $r_{2}+d r_{2}$, or is the probability density of finding an electron at a radius $r_{1}$ and another electron at $r_{2}$ simultaneously. This function is related by other function is called the two-particle radial density distribution function, that could tell us about how the different movements of the two electron as a result of mutual influence between them, as well as in the stabilization of one electron and noticing the change in the conduct of the another. And can be used in the calculation of the one-electron radial distribution function $\mathrm{D}\left(\mathrm{r}_{1}\right)$. The two-particle radial density distribution function $\mathrm{D}\left(r_{1}, r_{2}\right)$ is defined as [6].

$$
D\left(r_{1}, r_{2}\right)=\left(\begin{array}{c}
N \\
2
\end{array}\right) r_{1}^{2} r_{2}^{2} \int\left|\psi\left(x_{1}, \ldots, x_{N}\right)\right|^{2} d x_{3}, \ldots, d x_{N} d \Omega_{1} d \Omega_{2}
$$


Where $\left(\boldsymbol{r}_{i}, \Omega_{i}\right)$ is the polar coordinate of the vector $r_{i}$, it can be written $D\left(r_{1}, r_{2}\right)$ as [7]

$$
D\left(r_{1}, r_{2}\right)=\iint \Gamma\left(r_{1}, r_{2}\right) r_{1}^{2} r_{2}^{2} d \Omega_{1} d \Omega_{2}
$$

Where $\Gamma\left(r_{1}, r_{2}\right)$ is define two-particle density, by integrate $D\left(r_{1}, r_{2}\right)$ over the second electron we get of the one-electron radial density distribution function $\mathrm{D}\left(\mathrm{r}_{1}\right)$ represents the probability density function of finding an electron at a distance $r$ from the coordinate origin, i.e., the nucleus.

The usefulness from the expense the oneelectron radial density distribution function $\mathrm{D}\left(\mathrm{r}_{1}\right)$ in the determination of the expectation value for oneelectron $\left\langle r_{1}^{n}\right\rangle$.

$$
\left\langle r_{1}^{n}\right\rangle=\int_{0}^{\infty} \mathrm{D}\left(\mathrm{r}_{1}\right) r_{1}^{n} d r_{1}
$$

A measure of the spread or diffuseness of the one-particle radial density distribution function $\mathrm{D}\left(\mathrm{r}_{1}\right)$, may be obtained by evaluating $\Delta r_{1}$, the rootmean-square deviation is defined as [8].

$$
\begin{aligned}
\Delta r_{1}=\left[\left\langle r_{1}^{2}\right\rangle-\left\langle r_{1}\right\rangle^{2}\right]^{1 / 2} & (4) \\
f\left(r_{12}\right)=0.5 * r_{12} & {\left[\begin{array}{c}
\int_{0}^{r_{12}} r_{1} d r_{1} \int_{r_{12}-r_{1}}^{r_{12}+r_{1}} R_{1 s}^{2}\left(r_{1}\right) R_{1 s}^{2}\left(r_{2}\right) r_{2} d r_{2} \\
+ \\
\int_{r_{12}}^{\infty} r_{1} d r_{1} \int_{r_{1}-r_{12}}^{r_{1}+r_{12}} R_{1 s}^{2}\left(r_{1}\right) R_{1 s}^{2}\left(r_{2}\right) r_{2} d r_{2}
\end{array}\right] }
\end{aligned}
$$

Another function related with $\Gamma\left(r_{1}, r_{2}\right)$ is the radial electron-electron distribution function, which describes the probability of locating two electrons separated by distance $r_{12}$ from each other, was first introduced by Coulson and Neilson in their study of electron correlation for $\mathrm{He}\left({ }^{1} \mathrm{~S}\right)$ in the ground state. For atoms with more than two electrons, the radial electron-electron distribution function has a very convenient and unambiguous formulation in terms of the two-particle density matrix [9]:

$$
f\left(r_{12}\right)=\int \Gamma\left(r_{1}, r_{2}\right) d r_{1} d r_{2} / d r_{12}
$$

$f\left(r_{12}\right)$ representing a function of the distribution of the distance between electron 1 and electron 2 (this quantity fully determines the expectation value of the electronic Coulomb repulsion). It can be used in the calculation of the inter-particle expectation value $\left\langle r_{12}^{n}\right\rangle$ for two-particle, and used for the calculation Fermi hole and coulomb hole, $r_{12}$ is the inter-particle distance $r_{12}=\left|r_{1}-r_{2}\right|$. The inter-particle distribution function $f\left(r_{12}\right)$ is defined as[10],
The inter-particle expectation value $\left\langle r_{12}^{n}\right\rangle$ is given by the relation [11],

$$
\left\langle r_{12}^{n}\right\rangle=\int_{0}^{\infty} f\left(r_{12}\right) r_{12}^{n} d r_{12}
$$

The spread or diffusion of the inter-particle distribution function $f\left(r_{12}\right)$ about the mean value $\left\langle r_{12}^{n}\right\rangle$ is given by the standard deviation $\Delta r_{12}$ which is defined as

$$
\Delta r_{12}=\left[\left\langle r_{12}^{2}\right\rangle-\left\langle r_{12}\right\rangle^{2}\right]^{1 / 2}
$$

$\Delta r_{12}$ will determine the diffuse of the electronelectron distribution function $f\left(r_{12}\right)$ for each shell.

The total energy $\langle E\rangle$ corresponds to the expectation value of the Hamiltonian with respect to $\psi$ given by [13]

$\langle E\rangle=\left\langle\psi\left|\sum_{i=1}^{N}\left(-\frac{1}{2} \vec{\nabla}_{i}^{2}-\frac{\mathrm{z}}{\mathrm{r}_{\mathrm{i}}}\right)\right| \psi\right\rangle+\left\langle\psi\left|\sum_{i>j}^{N} \frac{1}{r_{i j}}\right| \psi\right\rangle(9)$

Notice that [14]:

$$
\langle T\rangle=-1 / 2 \int \psi^{*}\left[\sum_{i}^{N} \nabla_{i}^{2}\right] \psi d \tau
$$

$$
\langle V\rangle=\int \psi^{*}\left[\sum_{i}^{N}-\frac{Z}{r_{i}}+\sum_{i>j}^{N} \frac{1}{r_{i j}}\right] \psi d \tau
$$

Where:

$$
\begin{gathered}
<V_{e e}>=\int \psi^{*} \sum_{i>j}^{N} \frac{1}{\left|r_{i}-r_{j}\right|} \psi d \tau \\
<V_{e n}>=\int \psi^{*} \sum_{i}^{N}-\frac{Z}{r_{i}} \psi d \tau
\end{gathered}
$$

If an $\mathrm{N}$-electron atomic wave function satisfies the viral theorem then the energy expectation value is related to the potential energy by

$$
\langle E\rangle=\frac{1}{2}\langle V\rangle
$$

\section{Results and discussion}

Table 1 show that the results of one-particle expectation values for different powers ( $\mathrm{n}=-2$ to 2 ) in addition to calculate the standard deviation for intra shells $\mathrm{K}_{1 \mathrm{~s} \alpha} \mathrm{K}_{1 \mathrm{~s} \beta}, \mathrm{L}_{2 \mathrm{~s} \alpha} \mathrm{L}_{2 \mathrm{~s} \beta}$ and $\mathrm{L}_{2 \mathrm{p} \alpha}$, by comparing these results with published papers have been obtain good agreement. From this table, we noted effect the increase in atomic number with fixed the number of electrons, where observed when $\mathrm{n}$ takes negative values the expectation values of $\left\langle r_{1}^{n}\right\rangle$ increase with icreasing the atomic number for each shell, the values of $\left\langle r_{1}^{-1}\right\rangle$ refer to attractive energy between the nucleus and the electron, while 
when $\mathrm{n}$ takes positive values the expectation values of $\left\langle r_{1}^{n}\right\rangle$ are decreases because the distance between the electron and the nucleus become smallest as nuclear charge increases,also noted that the values of $\left\langle r_{1}\right\rangle \mathrm{K}_{1 \mathrm{~s} \alpha} \mathrm{K}_{1 \mathrm{~s} \beta}<\left\langle r_{1}\right\rangle \mathrm{L}_{2 \mathrm{~s} \alpha} \mathrm{L}_{2 \mathrm{~s} \beta}<\left\langle r_{1}\right\rangle \mathrm{L}_{2 \mathrm{p} \alpha}$ for all systems because the attractive energy for $\mathrm{K}_{1 \mathrm{~s} \alpha} \mathrm{K} 1 \mathrm{~s} \beta$ is largest from another shells. The values of standard deviation are decreases as atomic number increase because it depend on the values of $\left\langle r_{1}\right\rangle$ and $\left\langle r_{1}^{2}\right\rangle$.The results of inter-particle expectation values $\left\langle r_{12}^{n}\right\rangle$ and standard deviation are tabulated in table 2 for intra and inter shells, from these results we observed that the expectation values $\left\langle r_{12}^{n}\right\rangle \mathrm{K}_{1 \mathrm{~s} \alpha}$ $\mathrm{L}_{2 \mathrm{~s} \alpha} \equiv \mathrm{K}_{1 \mathrm{~s} \beta} \mathrm{L}_{2 \mathrm{~s} \beta}, \mathrm{K}_{1 \mathrm{~s} \beta} \mathrm{L}_{2 \mathrm{~s} \alpha} \equiv \mathrm{K}_{1 \mathrm{~s} \alpha} \mathrm{L}_{2 \mathrm{~s} \beta}, \mathrm{k}_{1 \mathrm{~S} \alpha} \mathrm{L}_{2 \mathrm{p} \alpha} \equiv \mathrm{k}_{1 \mathrm{~s} \beta} \mathrm{L}_{2 \mathrm{p} \alpha}$ and $\mathrm{L}_{2 \mathrm{~s} \alpha} \mathrm{L}_{2 \mathrm{p} \alpha} \equiv \mathrm{L}_{2 \mathrm{~s} \beta} \mathrm{L}_{2 \mathrm{p} \alpha}$, also we noted when $\mathrm{n}$ takes negative values the expectation values $\left\langle r_{12}^{n}\right\rangle$ increase as atomic number increase for all intra and inter shells where $\left\langle r_{12}^{-1}\right\rangle$ represented the repulsion energy between electrons, while when $\mathrm{n}$ takes positive values the $\left\langle r_{12}^{n}\right\rangle$ decreases also standard deviation decreases for all intra and inter shells and $\left\langle r_{12}\right\rangle \mathrm{K}_{1 \mathrm{~s} \alpha}$ $\mathrm{K}_{1 \mathrm{~s} \beta}<\left\langle r_{12}\right\rangle \mathrm{L}_{2 \mathrm{~s} \alpha} \mathrm{L}_{2 \mathrm{~s} \beta}$ because $\mathrm{K}$ shell is nearest to the nucleus conquently the attractive energy increase and lead to the distance between two electron in this shell become smaller. Table 3 show the expectation vales of all energies of the studied systems, from these results we observed all the energies increase as atomic number increase, $\mathrm{K}$ shell have largest contribution in the total energy of the system , by comparing the results of total energy of the systems with published papers have been obtain good agreement. Figure 1 show that the relation between the one-electron radial density distribution function $\mathrm{D}\left(\mathrm{r}_{1}\right)$ with the position for $1 \mathrm{~s}, 2 \mathrm{~s}$ and $2 \mathrm{p}$ shells respectively, where noted for each shell the maximum values of $\mathrm{D}(\mathrm{r} 1)$ increase when atomic number increase and location of these peaks contracted to ward of the nucleus, also observed the value of $\mathrm{D}(\mathrm{r} 1)$ vanishes when the distance equal zero or infinity, in the figure (1B) noted two peaks, the first represent the probability of finding the electron in small distance from the nucleus and the second represent the probability of finding the electron in $2 \mathrm{~s}$ shell. Figure 2 represented the relation between the radial electron-electron distribution function with the distance between two electrons for intra and inter shells where noted this function have the same behavior of $\mathrm{D}(\mathrm{r} 1)$ but observed in figure(2C) small peak at small distance, this results from Fermi hole .

Results of one-particle expectation values and the standard deviation.

Table 1

\begin{tabular}{|c|c|c|c|c|c|c|}
\hline System & Shell & $\left\langle r_{1}^{-2}\right\rangle$ & $\left\langle r_{1}^{-1}\right\rangle$ & $\left\langle r_{1}\right\rangle$ & $\left\langle r_{1}^{2}\right\rangle$ & $\Delta r 1$ \\
\hline \multirow{6}{*}{ B } & $\mathrm{K}_{1 \mathrm{~s} \alpha} \mathrm{K}_{1 \mathrm{~s} \beta}$ & 44.538805 & 4.67439 & 0.32587 & 0.14336 & 0.19280 \\
\hline & Ref.[15] & 44.53802 & 4.67433 & 0.32586 & 0.14336 & \\
\hline & $\mathrm{L}_{2 \mathrm{~s} \alpha} \mathrm{L}_{2 \mathrm{~s} \beta}$ & 2.02448 & 0.71288 & 1.97706 & 4.70913 & 0.89462 \\
\hline & Ref.[1] & 2.02447 & 0.71288 & 1.977064 & 4.70912 & \\
\hline & $\mathrm{L}_{2 p \alpha}$ & 0.52988 & 0.605007 & 2.20475 & 6.14607 & 1.13362 \\
\hline & Ref.[15] & 0.52988 & 0.605005 & 2.20475 & 6.14609 & \\
\hline \multirow{3}{*}{$\mathrm{C}^{+1}$} & $\mathrm{~K}_{1 \mathrm{~s} \alpha} \mathrm{K}_{1 \mathrm{~s} \beta}$ & 65.30838 & 5.66861 & 0.26811 & 0.09688 & 0.15810 \\
\hline & $\mathrm{L}_{2 \mathrm{~s} \alpha} \mathrm{L}_{2 \mathrm{~s} \beta}$ & 3.71798 & 0.96118 & 1.47558 & 2.58986 & 0.65105 \\
\hline & $\mathrm{L}_{2 \mathrm{p} \alpha}$ & 1.10361 & 0.88538 & 1.47873 & 2.68530 & 0.72063 \\
\hline \multirow{3}{*}{$\mathrm{N}^{+2}$} & $\mathrm{~K}_{1 \mathrm{~s} \alpha} \mathrm{K}_{1 \mathrm{~s} \beta}$ & 90.66488 & 6.66398 & 0.22767 & 0.06977 & 0.13392 \\
\hline & $\mathrm{L}_{2 s \alpha} \mathrm{L}_{2 \mathrm{~s} \beta}$ & 5.93078 & 1.21136 & 1.17884 & 1.64273 & 0.50305 \\
\hline & $\mathrm{L}_{2 \mathrm{p} \alpha}$ & 1.84150 & 1.15110 & 1.190578 & 1.54417 & 0.53709 \\
\hline
\end{tabular}

Table 2

Results of inter -particles expectation values and the standard deviation.

\begin{tabular}{|c|c|c|c|c|c|c|}
\hline System & Shell & $\left\langle r_{12}{ }^{-2}\right\rangle$ & $\left\langle r_{12}^{-1}\right\rangle$ & $\left\langle r_{12}\right\rangle$ & $\left\langle r_{12}^{2}\right\rangle$ & $\Delta r_{12}$ \\
\hline \multirow{6}{*}{ B } & $\mathrm{K}_{1 \mathrm{~s} \alpha} \mathrm{K}_{1 \mathrm{~s} \beta}$ & 14.40483 & 2.89205 & 0.47651 & 0.28672 & 0.24425 \\
\hline & $\mathrm{K}_{1 \mathrm{~s} \alpha} \mathrm{L}_{2 \mathrm{~s} \alpha} \equiv \mathrm{K}_{1 \mathrm{~s} \beta} \mathrm{L}_{2 \mathrm{~s} \beta}$ & 0.47459 & 0.61005 & 2.01279 & 4.85249 & 0.89507 \\
\hline & $\mathrm{K}_{1 \mathrm{~s} \beta} \mathrm{L}_{2 \mathrm{~s} \alpha}=\mathrm{K}_{1 \mathrm{~s} \alpha} \mathrm{L}_{2 \mathrm{~s} \beta}$ & 0.83853 & 0.64863 & 2.00848 & 4.85249 & 0.90470 \\
\hline & $\mathrm{k}_{1 \mathrm{~S} \alpha} \mathrm{L}_{2 \mathrm{p} \alpha} \equiv \mathrm{k}_{1 \mathrm{~s} \beta} \mathrm{L}_{2 \mathrm{p} \alpha}$ & 0.55863 & 0.60013 & 2.23350 & 6.28944 & 1.14057 \\
\hline & $\mathrm{L}_{2 \mathrm{~s} \alpha} \mathrm{L}_{2 \mathrm{~s} \beta}$ & 0.34455 & 0.46026 & 2.80505 & 9.41825 & 1.24496 \\
\hline & $\mathrm{L}_{2 s \alpha} \mathrm{L}_{2 p \alpha} \equiv \mathrm{L}_{2 s \beta} \mathrm{L}_{2 p \alpha}$ & 0.30677 & 0.43734 & 2.99031 & 10.25520 & 1.14596 \\
\hline \multirow{2}{*}{$\mathrm{C}^{+1}$} & $\mathrm{~K}_{1 \mathrm{~s} \alpha} \mathrm{K}_{1 \mathrm{~s} \beta}$ & 21.22107 & 3.51266 & 0.39190 & 0.19376 & 0.20043 \\
\hline & $\mathrm{K}_{1 \mathrm{~s} \alpha} \mathrm{L}_{2 \mathrm{~s} \alpha} \equiv \mathrm{K}_{1 \mathrm{~s} \beta} \mathrm{L}_{2 \mathrm{~s} \beta}$ & 0.81242 & 0.80316 & 1.50821 & 2.68675 & 0.64191 \\
\hline
\end{tabular}




\begin{tabular}{|l|l|l|l|l|l|l|}
\hline \multirow{7}{*}{} & $\mathrm{K}_{1 s \beta} \mathrm{L}_{2 s \alpha} \equiv \mathrm{K}_{1 s \alpha} \mathrm{L}_{2 s \beta}$ & 1.49034 & 0.8619 & 1.50386 & 2.68675 & $\mathbf{0 . 6 5 2 0 3}$ \\
\cline { 2 - 7 } & $\mathrm{k}_{1 \mathrm{~s} \alpha} \mathrm{L}_{2 p \alpha} \equiv \mathrm{k}_{1 s \beta} \mathrm{L}_{2 p \alpha}$ & 1.16832 & 0.87517 & 1.50009 & 2.78220 & $\mathbf{0 . 7 2 3 3 5}$ \\
\cline { 2 - 7 } & $\mathrm{L}_{2 s \alpha} \mathrm{L}_{2 s \beta}$ & 0.61516 & 0.61462 & 2.08769 & 5.17972 & $\mathbf{0 . 9 0 6 2 3}$ \\
\cline { 2 - 7 } & $\mathrm{L}_{2 s \alpha} \mathrm{L}_{2 p \alpha}=\mathrm{L}_{2 s \beta} \mathrm{L}_{2 p \alpha}$ & 0.60127 & 0.61640 & 2.09654 & 5.27517 & $\mathbf{0 . 9 3 7 9 1}$ \\
\hline \multirow{6}{*}{$\mathrm{N}^{+2}$} & $\mathrm{~K}_{1 s \alpha} \mathrm{K}_{1 s \beta}$ & 29.36503 & 4.13413 & 0.33269 & 0.13953 & $\mathbf{0 . 1 6 9 8 4}$ \\
\cline { 2 - 7 } & $\mathrm{K}_{1 s \alpha} \mathrm{L}_{2 s \alpha} \equiv \mathrm{K}_{1 s \beta} \mathrm{L}_{2 s \beta}$ & 1.23876 & 0.99513 & 1.20848 & 1.71251 & $\mathbf{0 . 5 0 2 0 8}$ \\
\cline { 2 - 7 } & $\mathrm{K}_{1 s \beta} \mathrm{L}_{2 s \alpha} \equiv \mathrm{K}_{1 s \alpha} \mathrm{L}_{2 s \beta}$ & 2.32898 & 1.07495 & 1.20429 & 1.71251 & $\mathbf{0 . 5 1 2 0 5}$ \\
\cline { 2 - 7 } & $\mathrm{k}_{1 \mathrm{~s} \alpha} \mathrm{L}_{2 p \alpha} \equiv \mathrm{k}_{1 s \beta} \mathrm{L}_{2 p \alpha}$ & 1.95276 & 1.13491 & 1.14706 & 1.61393 & $\mathbf{0 . 5 4 6 0 6}$ \\
\cline { 2 - 7 } & $\mathrm{L}_{2 s \alpha} \mathrm{L}_{2 s \beta}$ & 0.96342 & 0.76806 & 1.66572 & 3.28547 & $\mathbf{0 . 7 1 4 7 3}$ \\
\cline { 2 - 6 } & $\mathrm{L}_{2 s \alpha} \mathrm{L}_{2 p \alpha} \equiv \mathrm{L}_{2 s \beta} \mathrm{L}_{2 p \alpha}$ & 0.97410 & 0.78706 & 1.63347 & 3.18691 & $\mathbf{0 . 7 2 0 1 9}$ \\
\hline
\end{tabular}

The expectation values of the energies for studied systems.

\begin{tabular}{|c|c|c|c|c|c|c|}
\hline System & Shell & $\left\langle V_{e e}\right\rangle$ & $\left\langle V_{e n}\right\rangle$ & $\langle V\rangle$ & $\langle T\rangle$ & $\langle E\rangle$ \\
\hline \multirow{12}{*}{ B } & $\mathrm{K}_{1 s \alpha} \mathrm{K}_{1 s \beta}$ & 2.89205 & -46.7434 & -43.85135 & 21.92567 & -21.92567 \\
\hline & $\mathrm{K}_{1 s \alpha} \mathrm{L}_{2 \mathrm{~s} \alpha} \equiv \mathrm{K}_{1 \mathrm{~s} \beta} \mathrm{L}_{2 \mathrm{~s} \beta}$ & 0.61005 & & 0.61005 & 0.30502 & 0.30502 \\
\hline & $K_{1 s \beta} L_{2 s \alpha} \equiv K_{1 s \alpha} L_{2 s \beta}$ & 0.64863 & & 0.64863 & 0.32431 & 0.32431 \\
\hline & $\mathrm{k}_{1 \mathrm{~S} \alpha} \mathrm{L}_{2 \mathrm{p} \alpha} \equiv \mathrm{k}_{1 \mathrm{~s} \beta} \mathrm{L}_{2 \mathrm{p} \alpha}$ & 0.60013 & & 0.60013 & 0.30006 & 0.30006 \\
\hline & $\mathrm{L}_{2 s \alpha} \mathrm{L}_{2 s \beta}$ & 0.46026 & -7.1288 & -6.66854 & 3.33427 & -3.33427 \\
\hline & $\mathrm{L}_{2 s \alpha} \mathrm{L}_{2 p a}$ & 0.43734 & & 0.43734 & 0.21867 & 0.21867 \\
\hline & $\mathrm{L}_{2 s \beta} \mathrm{L}_{2 \mathrm{p} \alpha}$ & 0.43734 & -3.025 & -2.58766 & 1.29383 & -1.29383 \\
\hline & Total & 7.96441 & 56.8972 & -48.95259 & 24.47629 & -24.47629 \\
\hline & Ref.[16] & & & & & -24.53360 \\
\hline & Ref.[15] & & & -49.05812 & 24.52906 & -24.52906 \\
\hline & Ref.[17] & . & & & 24.52877 & -24.52877 \\
\hline & Ref.[1] & & & -49.05812 & 24.52906 & -24.52906 \\
\hline \multirow{9}{*}{$\mathrm{C}^{+1}$} & $\mathrm{~K}_{1 s \alpha} \mathrm{K}_{1 s \beta}$ & 3.51265 & -68.02332 & -64.51067 & 32.25533 & -32.25332 \\
\hline & $\mathrm{K}_{1 s \alpha} \mathrm{L}_{2 \mathrm{s \alpha}} \equiv \mathrm{K}_{1 \mathrm{~s} \beta} \mathrm{L}_{2 \mathrm{~s} \beta}$ & 0.80366 & & 0.80316 & 0.40152 & 0.40125 \\
\hline & $\mathrm{K}_{1 s \beta} \mathrm{L}_{2 \mathrm{s \alpha}} \equiv \mathrm{K}_{1 \mathrm{ss} \alpha} \mathrm{L}_{2 \mathrm{~s} \beta}$ & 0.8619 & & 0.8619 & 0.43095 & 0.43095 \\
\hline & $\mathrm{k}_{1 \mathrm{~S} \alpha} \mathrm{L}_{2 \mathrm{p} \alpha} \equiv \mathrm{k}_{1 \mathrm{~s} \beta} \mathrm{L}_{2 \mathrm{p} \alpha}$ & 0.87517 & & 0.87517 & 0.43758 & 0.43758 \\
\hline & $\mathrm{L}_{2 \mathrm{~s} \alpha} \mathrm{L}_{2 s \beta}$ & 0.61462 & -11.53416 & -10.91954 & 5.45977 & $-\mathbf{5 . 5 4 9 7 7}$ \\
\hline & $\mathrm{L}_{2 \mathrm{~s} \alpha} \mathrm{L}_{2 \mathrm{p} \alpha}$ & 0.61640 & & 0.61640 & 0.30820 & 0.30802 \\
\hline & $\mathrm{L}_{2 s \beta} \mathrm{L}_{2 \mathrm{p} \alpha}$ & 0.61640 & -5.31231 & -4.69591 & 2.34795 & -2.34795 \\
\hline & Total & 10.8679 & -85.09716 & -74.42926 & 37.21463 & -37.21463 \\
\hline & Ref.[18] & - & - & -74.58434 & 37.29221 & -37.29221 \\
\hline \multirow{9}{*}{$\mathrm{N}^{+2}$} & $\mathrm{~K}_{1 s \alpha} \mathrm{K}_{1 s \beta}$ & 4.13413 & -93.32968 & -89.16189 & 44.58079 & -44.58079 \\
\hline & $\mathrm{K}_{1 \mathrm{s \alpha} \alpha} \mathrm{L}_{2 \mathrm{s \alpha}} \equiv \mathrm{K}_{1 \mathrm{s \beta} \beta} \mathrm{L}_{2 \mathrm{~s} \beta}$ & 0.99513 & - & 0.99513 & 0.49756 & 0.49756 \\
\hline & $\mathrm{K}_{1 s \beta} \mathrm{L}_{2 \mathrm{~s} \alpha} \equiv \mathrm{K}_{1 \mathrm{ss} \alpha} \mathrm{L}_{2 \mathrm{~s} \beta}$ & 1.07495 & - & 1.07495 & 0.53747 & 0.53747 \\
\hline & $\mathrm{k}_{1 \mathrm{~S} \alpha} \mathrm{L}_{2 \mathrm{p} \alpha} \equiv \mathrm{k}_{1 \mathrm{~s} \beta} \mathrm{L}_{2 \mathrm{p} \alpha}$ & 1.13491 & - & 1.13491 & 0.56745 & 0.56745 \\
\hline & $\mathrm{L}_{2 s \alpha} \mathrm{L}_{2 s \beta}$ & 0.76806 & -16.95904 & -16.19098 & 8.09549 & -8.09549 \\
\hline & $\mathrm{L}_{2 s \alpha} \mathrm{L}_{2 p a}$ & 0.78706 & & 0.78706 & 0.39353 & 0.39353 \\
\hline & $\mathrm{L}_{2 s \beta} \mathrm{L}_{2 p a}$ & 0.78706 & -8.0577 & -7.27064 & 3.63532 & -3.63532 \\
\hline & Total & 12.92013 & -118.3463 & -105.4261 & 52.71349 & -52.71349 \\
\hline & Ref.[18] & & & -105.6321 & 52.81549 & -52.81549 \\
\hline
\end{tabular}




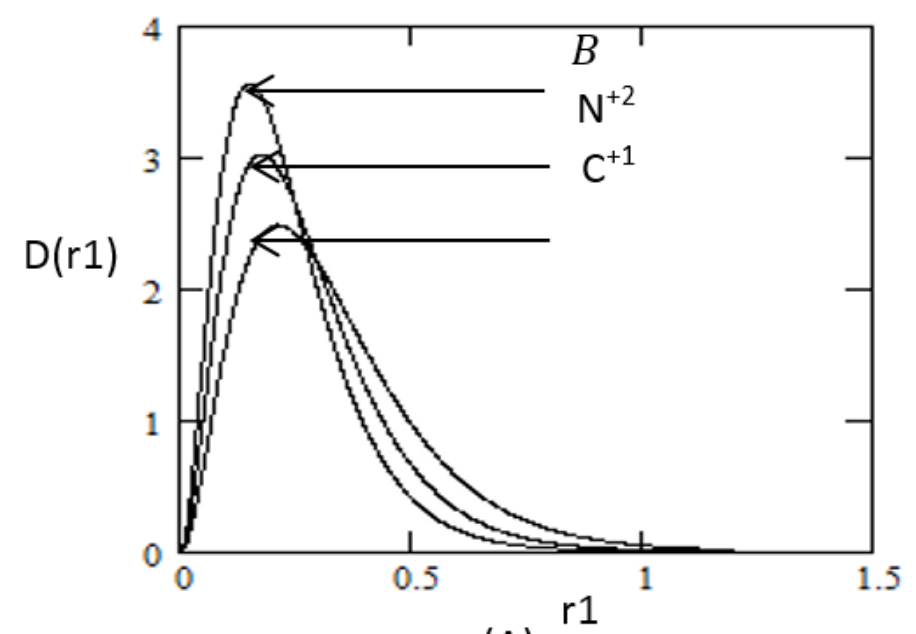

(A)
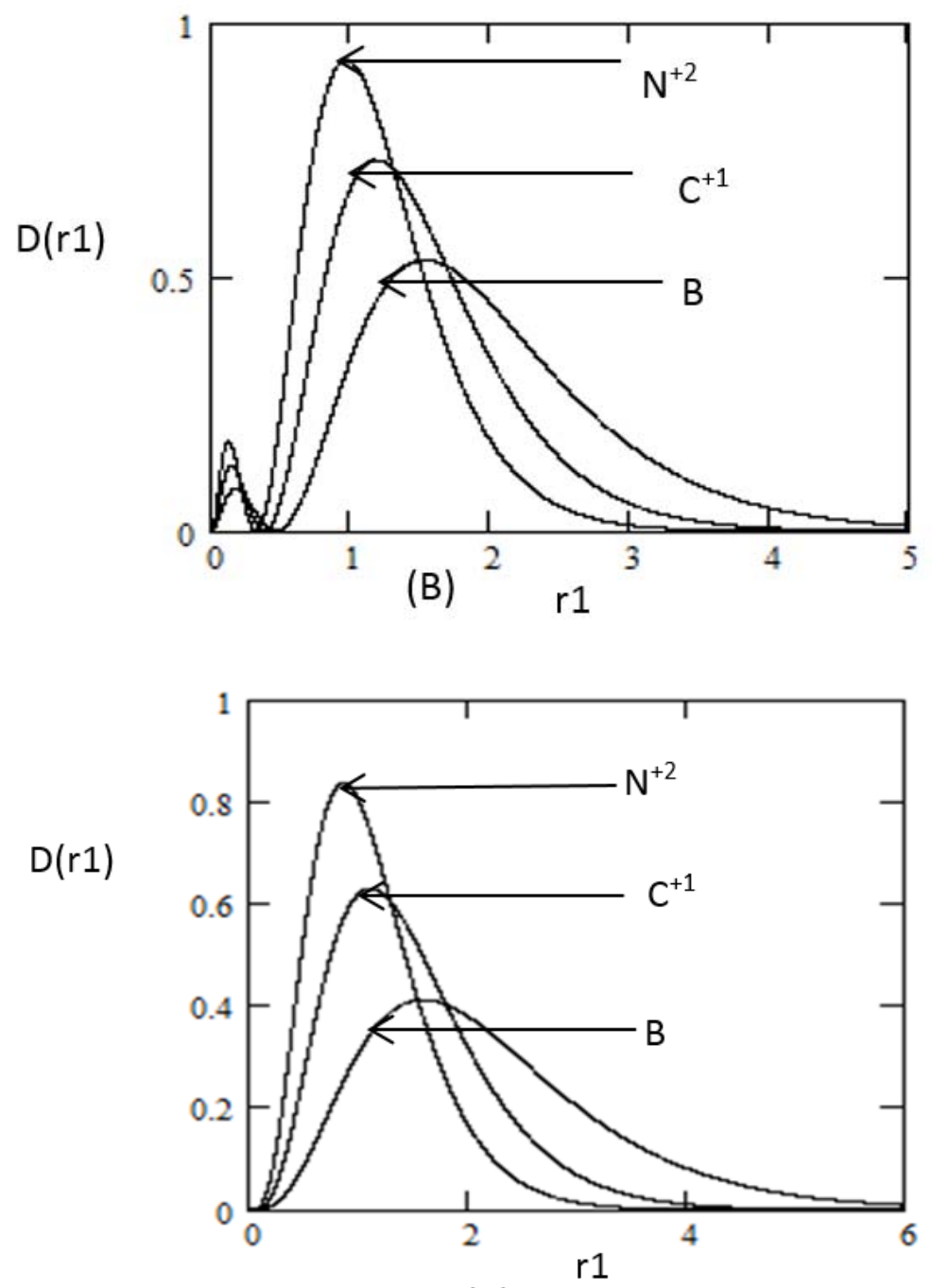

(C)

Figure 1 - The relation between one-electron radial density distribution function with the position

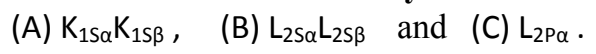




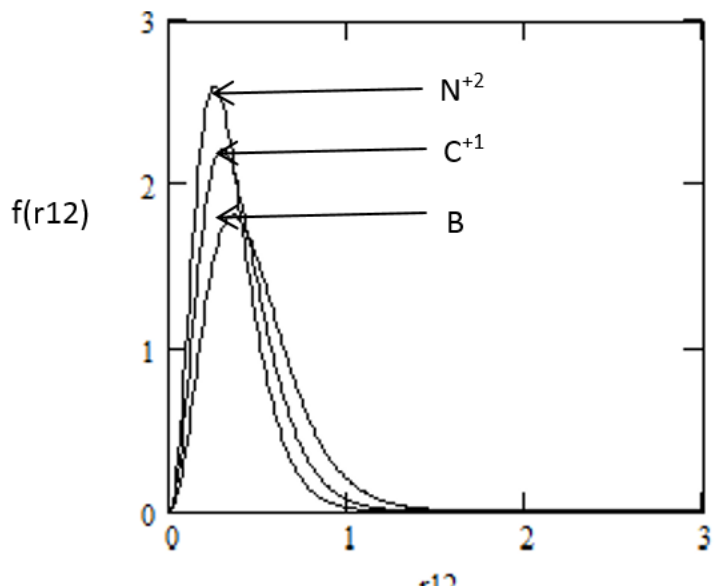

(A)

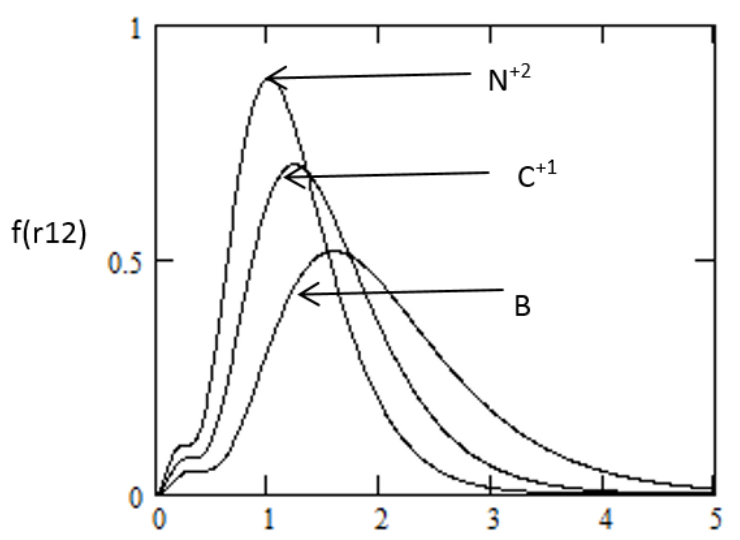

(C)

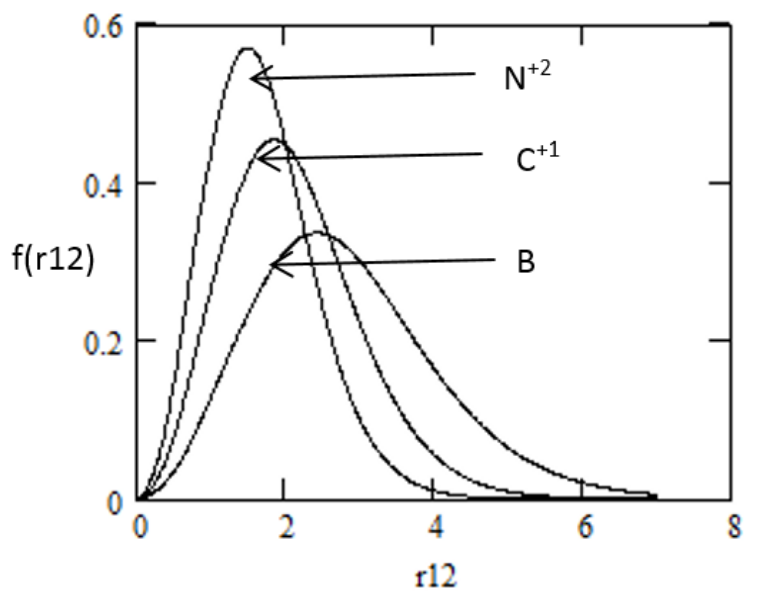

(E)

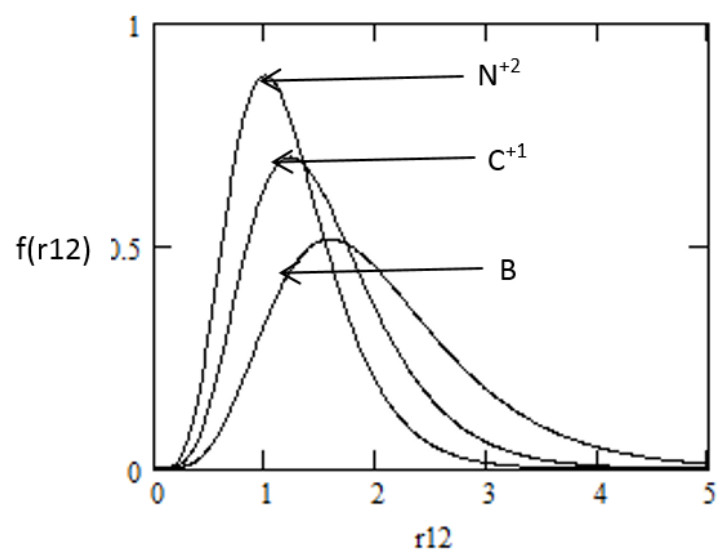

(B)

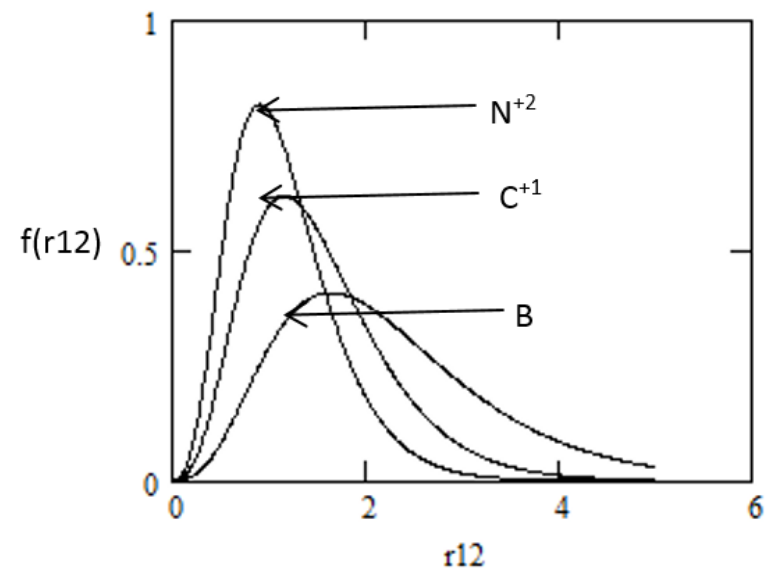

(D)

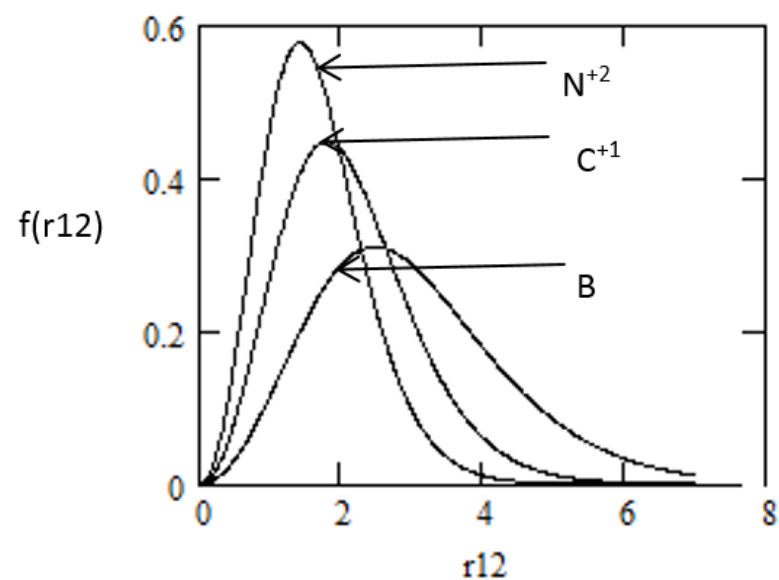

(F)

Figure 2 - The relation between the radial electron-electron distribution function with the distance between two electron

(A) $\mathrm{K}_{1 s \alpha} \mathrm{K}_{1 s \beta}$, (B) $\mathrm{K}_{1 s \alpha} \mathrm{L}_{2 s a} \equiv \mathrm{K}_{1 s \beta} \mathrm{L}_{2 s \beta}$, (C) $\mathrm{K}_{1 s \alpha} \mathrm{L}_{2 s \beta} \equiv \mathrm{K}_{1 s \beta} \mathrm{L}_{2 s \alpha}$,

(D) $\mathrm{K}_{1 s \alpha} \mathrm{L}_{2 \mathrm{P} \alpha} \equiv \mathrm{K}_{1 \mathrm{~s} \beta} \mathrm{L}_{2} \mathrm{P \alpha}$, (E) $\mathrm{L}_{2 s \alpha} \mathrm{L}_{2 s \beta}$ and (F) $\mathrm{L}_{2 s \alpha} \mathrm{L}_{2 \mathrm{P} \alpha} \equiv \mathrm{L}_{2 s \beta} \mathrm{L}_{2 \mathrm{P} \alpha}$. 


\section{Conclusions}

From these results can be conclude the expectation values of $\left\langle r_{1}^{n}\right\rangle$ and $\left\langle r_{12}^{n}\right\rangle$ inrease when $n$ takes negative values and the invers for positive values as atomic number increase while the expectation values of all energies are increase as nuclear charge increase and the results of total energy for studied systems agreement with the published papers, the maximum values of $\mathrm{D}(\mathrm{r} 1)$ and $\mathrm{f}(\mathrm{r} 12)$ increase and locations of these peaks decreases as atomic number increase

\section{References:}

1. Bunge C (1993) J. Atomic Data and Nuclear Data Tables, V.53(1993) 113-162.

2. Iqbal A (2012) M.Sc.Thesis "Some aspects on the Schrodinger equation", Chaimers University of technology.

3. Hill Q (2010) ph.D. Thesis "Development of more accurate computational methods within linear -scaling density functional theory", Southampton University.

4. Guseinov I, Aydin R, Mamedov B (2003) J. Mol Model, 9(2003) 325-328.

5. Beran GJ (2005) ph.D. Thesis "Fast electronic structure methods for studying highly correlated molecular systems .'University of California.

6. Matsuyama H, Koga T (2010) Computational and Applied Mathemtics, V.233(2010) 15841589.

7. Banyard KE, Baker CC (1969) J. Chemical Physical,V.51, No. 6,(1969) 2680-2689.

8. Toylor GK, Banyard KE (1973) J. Physical Review A, V.8, No.3(1973) 1157.

9. Boyd RJ, Main C.Yee (1982) J. Chem.Phys, V.77, No.7(1982) 3578-3582.
10. Giorgi PG, Savin A (2005) J. Physical Review A,V.71, 032513(2005) 1-9.

11. Gupta A, Russell J.Boyd (1978)J. Chem.Phys, V.68. No.4.

12. Simons J (1972) J. Quantum Chem, V.1, 439448.

13. Riveros AF, Contreras A (2008) J. Physics Letters A,V.372 (2008) 6175-6182.

14. Krebs S (1999) J. Computer physics Communications, V.116(1999) 137-277.

15. Saito SL (2009) J. Atomic Data and Nuclear Data table, V.95(2009)836-870.

16. Maldonado P, Sarsa A, Buendia E, Galvez FJ (2010) J. Atomic Data and Nuclear Data tables V.95 (2010) 1-6.

17. Sarsa A, Buendia E, Galvez FJ (2004) J.Atomic Data and Nuclear Data tables,V.95 (2004) 163-202.

18. Clement E, Roetti C (1974) J. Atomic Data and Nuclear Data Tables, V.14, No. 3-4(1974) 177478. 